\title{
High-Efficiency Synthesis and Properties of Latent Pigment Red 272DPP-BOC by Microwave Irradiation
}

\author{
Tomoji Ohishi1 ${ }^{*}$, Yu Kimura1, Kiyohiko Nakajima², Masayuki Watanabe3, Noboru Aoyagi ${ }^{3}$ \\ ${ }^{1}$ Department of Applied Chemistry, Faculty of Engineering, Shibaura Institute of Technology, Tokyo, Japan \\ ${ }^{2}$ Department of Chemistry, Aichi University of Education, Kariya, Japan \\ ${ }^{3}$ Nuclear Science and Engineering Center, Sector of Nuclear Science Research, Japan Atomic Energy Agency, Naka-gun, Ibaraki, \\ Japan \\ Email: ^tooishi@sic.shibaura-it.ac.jp
}

How to cite this paper: Ohishi, T., Kimura, Y., Nakajima, K., Watanabe, M. and Aoyagi, N. (2020) High-Efficiency Synthesis and Properties of Latent Pigment Red 272DPP-BOC by Microwave Irradiation. Materials Sciences and Applications, 11, 195-203.

https://doi.org/10.4236/msa.2020.113013

Received: February 10, 2020

Accepted: March 7, 2020

Published: March 10, 2020

Copyright $\odot 2020$ by author(s) and Scientific Research Publishing Inc. This work is licensed under the Creative Commons Attribution International License (CC BY 4.0).

http://creativecommons.org/licenses/by/4.0/

\begin{abstract}
A high-efficiency synthesis method for a latent pigment of red pigment diketo-pyrrolo-pyrrole (Pig. Red 272:272DPP), which is important as a functional organic pigment, was investigated, and the investigation results revealed that irradiation of microwaves (MWs) for several seconds to 272 DPP in NMP (N-methyl-2-pyrrolidone) solvent yielded DPP latent pigment (272DPP-BOC) at a high yield of $86.2 \%$. Two kinds of latent-pigment crystals, namely, red and yellow, were obtained by recrystallization, and it was found that the fluorescence-emission properties of the two kinds differ significantly. Single-crystal X-ray structural analysis showed that the difference in the fluorescence-emission properties of the two types is derived from the difference in their crystal structures.
\end{abstract}

\section{Keywords}

Latent Pigment, Diketopyrrolopyrrol, Pigment Red 272, High Efficiency Synthesis, Microwave Irradiation, Single-Crystal X-Ray Analysis

\section{Introduction}

Diketo-pyrrolo-pyrrole (DPP) is a very important functional organic pigment used as a red pigment for red color filter films in displays such as liquid crystal devices (LCDs) and organic electroluminescent (EL) devices known as OLEDs [1] [2]. Recently, it has attracted attention as a material for organic semiconductors [3] [4] [5], and it is considered to be an organic pigment of increasing importance in the future. Since an organic pigment is insoluble in various solvents, 
it is difficult to uniformly mix it in a solution and form a thin film. "Solubilization" of an organic pigment has therefore become a major development issue. "Latentizing" of an organic pigment is a means of introducing a releasable substituent into the pigment (which is insoluble in a solvent) in order to solubilize the pigment. Iqbal et al. reported that by introducing bulky t-butyl-carbonate ( $\mathrm{t}-\mathrm{BOC}$ ) into the -NH group of the DPP pigment "Pig. Red 255" made it possible to suppress the hydrogen bonds between molecules and $\pi-\pi$ stacking and thereby the pigment became soluble in a solvent [6]. They also reported that the formed latent pigment (255 DPP-BOC) was returned to the original pigment (DPP) by heat treatment. This property has attracted attention because it is easy to prepare micro or nanoparticles of DPP and form a thin film with them; consequently, the detailed chemical properties of Pig. Red 255-BOC have been investigated [7] [8]. However, although Pig. Red 272 is an important DPP analog, its latent pigmentation and properties have not been investigated in detail. In addition, the latent pigment is synthesized by stirring DPP with t-BOC in a solvent at room temperature for 24 to 36 hours. Shortening of the reaction time and increasing yield are therefore two key issues to be addressed.

In this study, a high-efficiency method for synthesizing the latent pigment Pig. Red 272 (272DPP-BOC) using microwave (MW) irradiation was developed, and the crystal structure and the fluorescence-emission characteristics of the synthesized 272DPP-BOC were investigated. The main findings of this investigation are summarized as follows: 1) MW irradiation to 272DPP in NMP solvent gives a high yield of 272DPP-BOC in a short time (several seconds); 2) yellow crystals and red crystals were obtained by recrystallization of 272DPP-BOC, and single-crystal X-ray analysis of both revealed that although they are both disubstituted with the same BOC group, their crystal structures differ; and 3) the yellow 272DPP-BOC crystal has stronger fluorescent properties.

\section{Experimental}

\subsection{Synthesis of 272DPP-BOC}

As a catalyst, $0.183 \mathrm{~g}(1.50 \mathrm{mmol})$ of 4-DMAP (4-dimethylamino pyridine; Tokyo Kasei Co.) and as the starting pigment, $0.158 \mathrm{~g}(0.500 \mathrm{mmol})$ of $272 \mathrm{DPP}$ (Dainichiseika Kogyo Co.) were dispersed in $10 \mathrm{~mL}$ of NMP solvent (N-methyl 2-pyrrolidone; Tokyo Kasei Co.), and the mixture was stirred. After that, $0.874 \mathrm{~g}$ $(4.00 \mathrm{mmol})$ of t-BOC reagent (di-t-butyl-di-carbonate; Tokyo Kasei Co.) was added to the mixture. This solution was irradiated with $2.45-\mathrm{GHz}$ microwaves (MWs) at $100 \mathrm{~W}$ for different predetermined times. The reaction solution was added to $180 \mathrm{~mL}$ of pure water, stirred, and allowed to stand for 1 hour to form a yellow powder. Thereafter, the powder was suction filtrated and dried under reduced pressure to obtain crude crystals. The crystalline powder formed after drying under reduced pressure was dissolved with $30 \mathrm{~mL}$ of chloroform, and when hexane was added, yellow crystals were formed. Yields (\%) were calculated to divide the value $(\mathrm{g})$ of the obtained latent pigment yield by that $(\mathrm{g})$ of theoret- 
ical yield.

\subsection{Preparation of Yellow Crystals and Red Crystals}

Yellow and red crystals were obtained by two different kinds of recrystallization manner.

First, $1.1 \mathrm{~mL}$ of chloroform was added to and dissolved in $0.1 \mathrm{~g}$ of 272 DPP-BOC (yellow crystals obtained in 2.1), and $1.1 \mathrm{~mL}$ of methanol was slowly added and left to stand for 24 hours, after which time, yellow crystals precipitated. The crystals were then suction filtered and dried under reduced pressure.

To prepare a saturated 272DPP-BOC solution, which is high-concentration solution of 272DPP-BOC, $1.1 \mathrm{~mL}$ of chloroform was added to and dissolved in $0.1 \mathrm{~g}$ of 272DPP-BOC. When the 272DPP-BOC chloroform solution was added to $7 \mathrm{~mL}$ of methanol under stirring, red crystals were promptly formed. The crystals were then suction filtered and dried under reduced pressure to obtain the red crystals.

\subsection{Evaluation}

The molecular structure of the latent pigment was analyzed by using a Fourier transform infrared spectrophotometer (Shimadzu, FTIR-8400S) with the $\mathrm{KBr}$ pellet method. And the single-crystal structure of the yellow and the red crystals was determined by using a single-crystal X-ray analyzer (Rigaku, R-AXIS RAPID).

Fluorescence-emission spectra were measured by using a fluorescent phosphorescent spectrophotometer (HORIBA Scientific, Fluoromax-4P) while irradiating incident light at a $45^{\circ}$ angle onto a cell containing a powder sample of the crystals. As an integrating sphere for the fluorescent phosphorescence spectrophotometer, a Quanta- $\varphi$ apparatus (HORIBA Scientific) was used for measuring absolute quantum yield.

\section{Results and Discussion}

\subsection{Synthesis of Latent Pigment by MW Irradiation}

In regard to the synthesis of the latent pigment 272DPP-BOC, a t-BOC group was substituted into the $-\mathrm{NH}$ group of the 272DPP by the reaction of the $\mathrm{t}-\mathrm{BOC}$ with the starting material, 272DPP (see Figure 1). It was found that this reaction proceeds very rapidly when 272DPP in the NMP solvent is irradiated with MWs. When irradiated with MW, the reaction proceeded immediately, and the color of the reaction solution changed from a red dispersion solution to a transparent yellow solution. The relationship between MW irradiation time and yield is plotted in Figure 2. According to the figure, the yield is the highest (82.6\%) when MW irradiation was applied for 6 seconds. When the reaction was promoted by stirring in NMP for 24 hours without MW irradiation, the yield was $63.8 \%$. It is noteworthy that the reaction time was markedly shortened by the MW irradiation, and a latent pigment could be obtained at high yield. When 


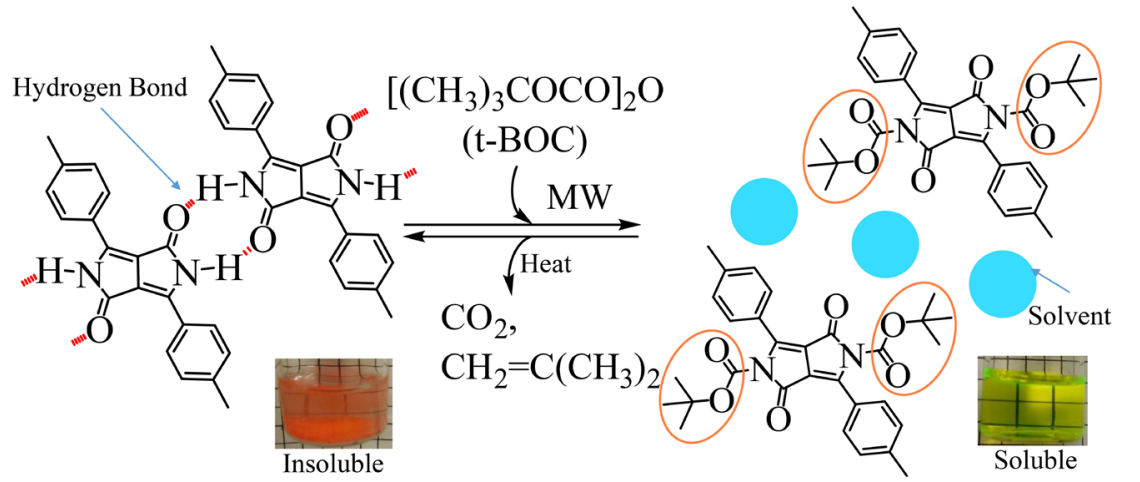

P.R.272 (272DPP)

* DPP:diketopyrrolo-pyrrole

Latent Pigment : 272DPP-BOC

Figure 1. Synthetic procedure of latent pigment 272DPP-BOC using MW irradiation.

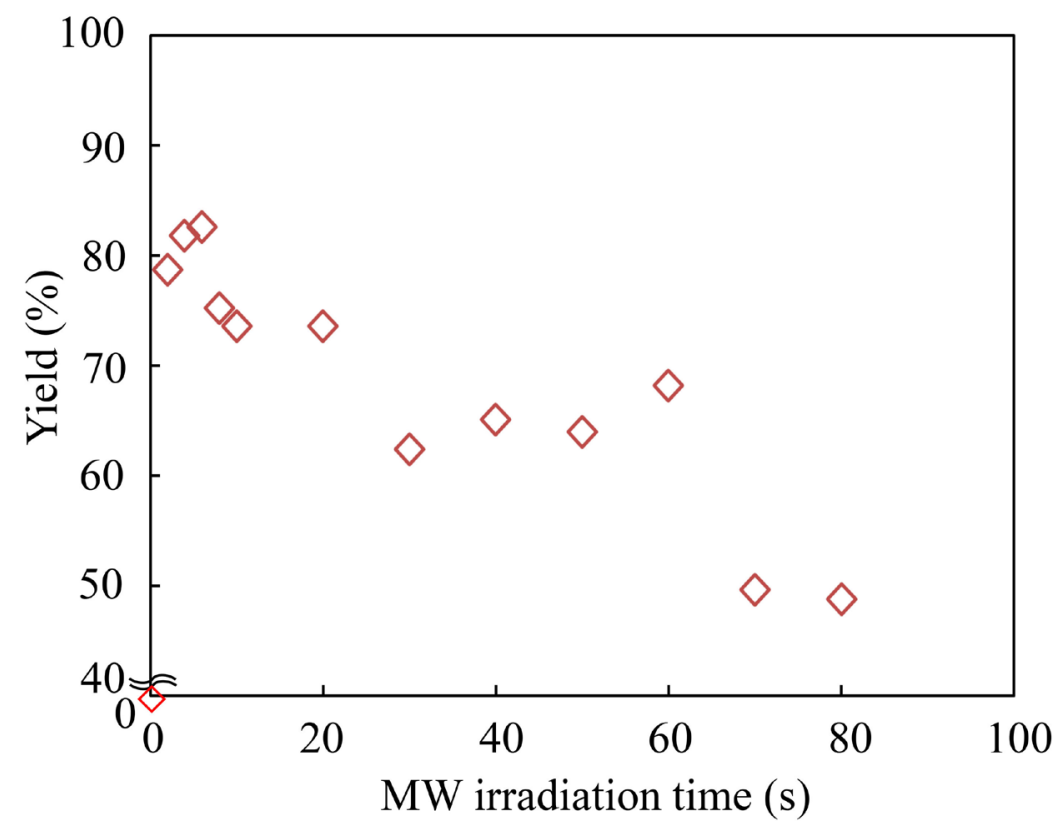

Figure 2. Relationship between yield of 272DPP-BOC and MW irradiation time.

MW irradiation time was extended, the yield decreased and reached about $49 \%$ at irradiation time of 80 seconds. From the reaction solution after 80 seconds, the starting pigment, namely, 272DPP, was detected. This result indicates that once the t-BOC group was substituted into the -NH group of the 272DPP, a latent pigment was formed; however, when the MW irradiation time was long, the substituted t-BOC was eliminated. When the irradiation time was long, the temperature of the NMP solvent also increased, namely, rising to $62^{\circ} \mathrm{C}$ in 30 seconds, and to almost $100^{\circ} \mathrm{C}$ in 80 seconds. It is supposed that the rise in the temperature of the solvent promotes elimination of the $\mathrm{t}$-BOC group in a reverse manner to the substitution reaction. The present state of affairs regarding the effect of MW irradiation on the reaction is not clear; even so, in consideration that almost no temperature rise of the NMP solvent is observed in the case of MW irradiation for several seconds, it seems that the MW irradiation acts directly on 
the 272DPP molecules (with polarity) in the NMP solvent, so the substitution reaction proceeds very rapidly.

When the yellow powder obtained by MW irradiation was recrystallized, 272DPP-BOC was dissolved in chloroform, methanol was added thereto, and the mixture was allowed to stand for 24 hours to obtain yellow crystals. On the other hand, when the chloroform solution was added in methanol, red crystals were obtained. The infrared absorption spectra of the yellow crystals and the red crystals are shown in Figure 3. For comparison, the spectra of the 272DPP starting pigment and spectra of yellow and red crystals heat-treated at $180^{\circ} \mathrm{C}$ for 30 minutes are also shown. Both (red and yellow crystals) spectra are very similar, and the $v \mathrm{~N}-\mathrm{H}$ vibration peaks in the vicinity of $3400 \mathrm{~cm}^{-1}$ present in spectrum of 272DPP is not present in the spectra of 272DPP-BOC yellow and red, and the $v C=O$ and $v C-O-C$ vibration peaks appear around $1600 \mathrm{~cm}^{-1}$ and 1200 $\mathrm{cm}^{-1}$, respectively. From this result, it is considered that the hydrogenatoms of 272DPP are replaced with t-BOC groups. When the yellow crystals and red crystals were heat-treated, a spectrum [(d) in Figure 3] similar to that of 272DPP was obtained. This result suggests that both t-BOC groups were eliminated by heating, thereby returning the 272DPP-BOC to 272DPP.

\subsection{Determination of Molecular Structure by Single-Crystal X-Ray Structural Analysis}

$\mathrm{X}$-ray structural analysis of single crystals was used to determine the molecular structure of the yellow crystals and the red crystals. The differences in the molecular structures and crystal structures of both 272DPP-BOC types are shown in Figure 4, and the crystal-structure data and bond distances/angles are listed in Table 1 and Table 2, respectively. These results confirm that both the yellow and the red crystals of 272DPP-BOC have two BOC groups substituted onto a

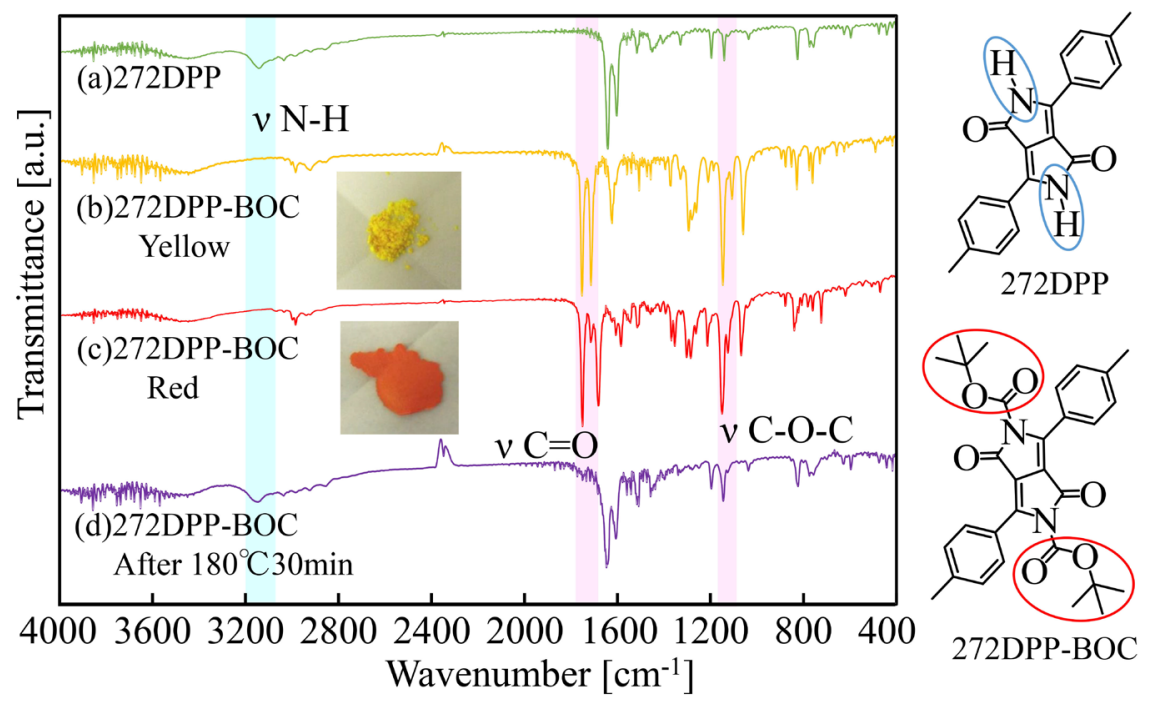

Figure 3. Infrared spectra of 272DPP-BOC. (a) 272DPP, (b) 272DPP-BOC Yellow, (c) 272DPP-BOC Red, (d) After heat treatment. 

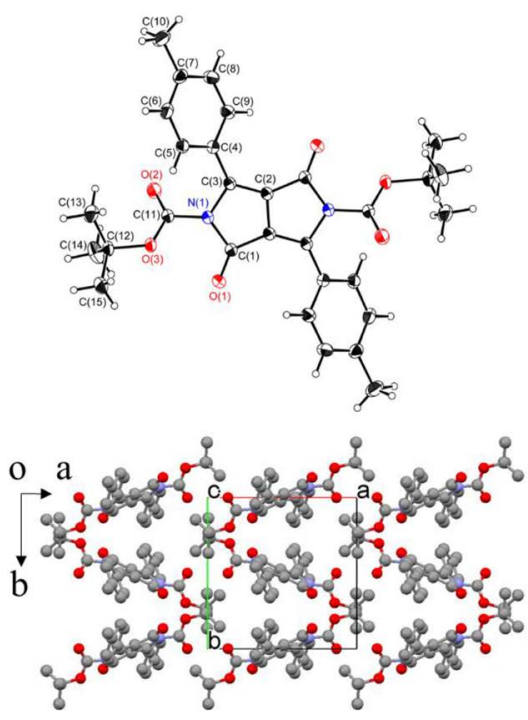

(a)
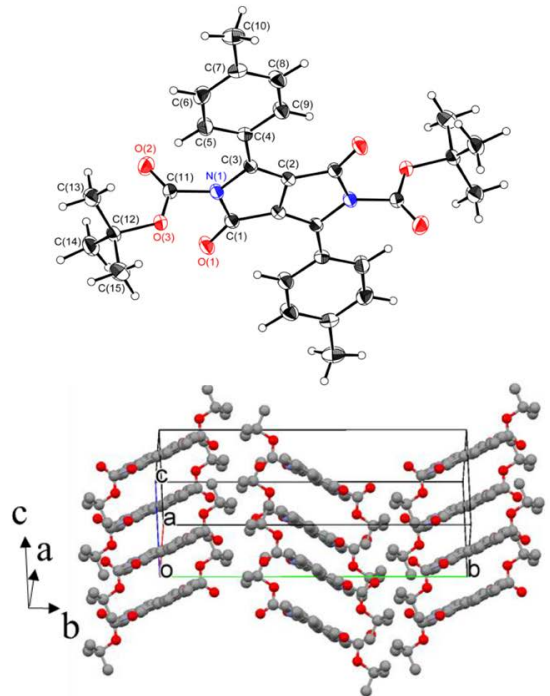

(b)

Figure 4. Crystal structure of 272DPP-BOC. (a) 272DPP-BOC Yellow, (b) 272DPP-BOC Red.

Table 1. Crystal structural data of 272DPP-BOC.

\begin{tabular}{ccc}
\hline Color & Yellow & Red \\
\hline Compositional formula & $\mathrm{C}_{30} \mathrm{H}_{32} \mathrm{~N}_{2} \mathrm{O}_{6}$ & $\mathrm{C}_{30} \mathrm{H}_{32} \mathrm{~N}_{2} \mathrm{O}_{6}$ \\
Crystal System & 516.58 & 516.58 \\
Space group & Monoclinic & Monoclinic \\
$a(\AA)$ & $P 12_{1} / c 1$ & $P 12_{1} / n 1$ \\
$b(\AA)$ & $10.488(6)$ & $6.308(2)$ \\
$c(\AA)$ & $10.587(4)$ & $22.948(9)$ \\
angle $\left(^{\circ}\right)$ & $12.266(6)$ & $9.122(4)$ \\
$V\left(\AA^{3}\right)$ & $\alpha=\gamma=90.00$ & $\alpha=\gamma=90.00$ \\
$Z$ & $\beta=95.28(2)$ & $\beta=91.502(19)$ \\
$T(\mathrm{~K})$ & $1356.2(11)$ & $1319.9(9)$ \\
$R$ & 2 & 2 \\
$w R$ & 298 & 298 \\
$\mathrm{GOF}$ & 0.0385 & 0.0418 \\
& 0.1127 & 1.095 \\
\hline
\end{tabular}

nitrogen atom. Moreover, it is clear that the yellow crystal and red crystal differ in terms of the ways the molecules are twisted and stacked. It is also clear that the crystal structures differ because the space group of the yellow crystal is $\mathrm{P} 12_{1} / \mathrm{c} 1$ and that of the red crystal is $\mathrm{P} 12_{1} / \mathrm{n} 1$. In the case of the red crystal, the benzene rings bonded to the DPP skeleton are arranged in parallel between DPP 
Table 2. Bond distances $(\AA)$ and angles $\left({ }^{\circ}\right)$.

\begin{tabular}{|c|c|c|c|}
\hline \multicolumn{2}{|c|}{ Yellow } & \multicolumn{2}{|c|}{ Red } \\
\hline 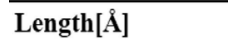 & Angle $\left[{ }^{\circ}\right]$ & Length $[\AA]]$ & Angle $\left[{ }^{\circ}\right]$ \\
\hline $\mathrm{O} 1 \mathrm{C} 11.2044(15)$ & C11 O3 C12 119.54(10) & O1 Cl 1.2175(14) & $\mathrm{C} 11 \mathrm{O} 3 \mathrm{C} 12$ 121.24(9) \\
\hline O2 C11 1.1891(15) & C3 N1 C11 122.04(10) & O2 C11 1.1887(15) & C3 N1 C1 112.70(9) \\
\hline O3 C11 1.3089(14) & C3 N1 C1 111.42(9) & O3 C11 $1.3057(16)$ & C3 N1 C11 130.75(10) \\
\hline O3 C12 1.4992(16) & C11 N1 C1 122.31(10) & O3 C12 1.4946(14) & C1 N1 C11 116.45(9) \\
\hline N1 C3 1.4134(15) & $\mathrm{O} 1 \mathrm{C} 1 \mathrm{~N} 1$ 124.33(11) & N1 C3 1.4001(14) & $\mathrm{O} 1 \mathrm{C} 1 \mathrm{~N} 1121.56(11)$ \\
\hline N1 C11 1.4332(16) & $\mathrm{O} 1 \mathrm{C} 1 \mathrm{C} 2133.06(11)$ & N1 C1 1.4206(15) & O1 C1 C2 134.84(11) \\
\hline N1 C1 1.4462(16) & $\mathrm{N} 1 \mathrm{C} 1 \mathrm{C} 2102.59(10)$ & N1 C11 $1.4418(15)$ & $\mathrm{N} 1 \mathrm{C} 1 \mathrm{C} 2103.59(9)$ \\
\hline $\mathrm{C} 1 \mathrm{C} 21.4543(17)$ & C3 C2 C2 109.92(13) & $\mathrm{C} 1 \mathrm{C} 21.4445(16)$ & C3 C2 C2 110.44(12) \\
\hline C2 C3 1.3648(17) & C3 C2 C1 141.13(11) & C2 C3 1.3839(16) & C3 C2 C1 141.69(10) \\
\hline $\mathrm{C} 2 \mathrm{C} 2$ 1.424(2) & $\mathrm{C} 2 \mathrm{C} 2 \mathrm{C} 1$ 108.93(13) & $\mathrm{C} 2 \mathrm{C} 21.420(2)$ & $\mathrm{C} 2 \mathrm{C} 2 \mathrm{C} 1107.87(12)$ \\
\hline $\mathrm{C} 2 \mathrm{C} 11.4543(17)$ & C2 C3 N1 107.01(10) & $\mathrm{C} 2 \mathrm{C} 11.4445(16)$ & C2 C3 N1 105.39(9) \\
\hline C3 C4 1.4561(18) & $\mathrm{C} 2 \mathrm{C} 3 \mathrm{C} 4130.82(11)$ & C3 C4 1.4545(16) & C2 C3 C4 129.98(10) \\
\hline C4 C5 1.3933(17) & N1 C3 C4 122.00(10) & C4 C5 $1.3943(18)$ & N1 C3 C4 124.62(10) \\
\hline C4 C9 1.3939(17) & C5 C4 C9 118.60(12) & C4 C9 1.3952(17) & C5 C4 C9 116.94(11) \\
\hline C5 C6 1.3814(19) & C5 C4 C3 121.47(11) & C5 C6 1.3831(19) & C5 C4 C3 124.17(11) \\
\hline C5 H1 0.979(15) & C9 C4 C3 119.92(11) & C5 H1 0.974(17) & C9 C4 C3 118.88(11) \\
\hline C6 C7 1.387(2) & C6 C5 C4 120.40(12) & C6 C7 1.375(2) & C6 C5 C4 121.10(12) \\
\hline C6 H2 0.948(17) & C6 C5 H1 120.8(9) & C6 H2 0.98(2) & C6 C5 H1 117.7(10) \\
\hline C7 C8 1.386(2) & C4 C5 H1 118.8(9) & C7 C8 1.386(2) & C4 C5 H1 121.2(10) \\
\hline $\mathrm{C} 7 \mathrm{C} 101.508(2)$ & C5 C6 C7 121.22(13) & C7 C10 1.5043(18) & C7 C6 C5 121.71(13) \\
\hline C8 C9 1.382(2) & C5 C6 H2 119.4(10) & C8 C9 1.3777(18) & C7 C6 H2 118.7(11) \\
\hline C8 H3 0.952(18) & C7 C6 H2 119.2(10) & C8 H3 0.980(18) & C5 C6 H2 119.6(11) \\
\hline C9 H4 0.987(17) & C8 C7 C6 118.07(12) & C9 H4 0.969(19) & C6 C7 C8 117.42(11) \\
\hline $\mathrm{C} 10 \mathrm{H} 51.02(5)$ & $\mathrm{C} 8 \mathrm{C} 7 \mathrm{C} 10$ 121.16(16) & $\mathrm{C} 10 \mathrm{H} 50.93(3)$ & C6 C7 C10 121.09(14) \\
\hline C10 H6 0.90(4) & C6 C7 C10 120.77(16) & C10 H6 0.94(3) & C8 C7 C10 121.48(14) \\
\hline $\mathrm{C} 10 \mathrm{H} 70.89(4)$ & C9 C8 C7 121.44(13) & $\mathrm{C} 10 \mathrm{H} 7 \mathrm{1.00(3)}$ & C9 C8 C7 121.61(12) \\
\hline $\mathrm{C} 12 \mathrm{C} 141.506(2)$ & C9 C8 H3 120.5(11) & $\mathrm{C} 12 \mathrm{C} 15$ 1.507(2) & C9 C8 H3 115.8(10) \\
\hline $\mathrm{C} 12 \mathrm{C} 15$ 1.509(2) & C7 C8 H3 118.0(11) & $\mathrm{C} 12 \mathrm{C} 141.507(2)$ & C7 C8 H3 122.6(10) \\
\hline $\mathrm{C} 12 \mathrm{C} 13$ 1.514(2) & C8 C9 C4 120.15(13) & C12 C13 1.5091(19) & C8 C9 C4 121.16(12) \\
\hline C13 H8 0.99(2) & C8 C9 H4 120.7(9) & $\mathrm{C} 13 \mathrm{H} 81.01(2)$ & C8 C9 H4 121.1(10) \\
\hline C13 H9 1.03(2) & C4 C9 H4 119.1(9) & C13 H9 0.97(2) & C4 C9 H4 117.7(10) \\
\hline C13 H10 1.01(3) & C7 C10 H5 113(2) & C13 H10 0.99(2) & C7 C10 H5 111.5(16) \\
\hline C14 H11 1.00(3) & C7 C10 H6 112(2) & C14 H11 0.98(2) & C7 C10 H6 109.5(13) \\
\hline C14 H12 1.00(3) & H5 C10 H6 115(3) & C14 H12 1.04(2) & H5 C10 H6 114(2) \\
\hline C14 H13 1.00(2) & C7 C10 H7 111(2) & C14 H13 0.98(2) & C7 C10 H7 112.6(14) \\
\hline C15 H14 0.93(2) & H5 C10 H7 94(3) & C15 H14 0.96(4) & H5 C10 H7 107(2) \\
\hline C15 H15 0.97(2) & $\mathrm{H} 6 \mathrm{C} 10 \mathrm{H} 7110(3)$ & C15 H15 1.01(3) & H6 C10 H7 101.8(18) \\
\hline C15 H16 1.03(2) & O2 C11 O3 $128.75(12)$ & C15 H16 0.97(3) & O2 C11 O3 $129.46(11)$ \\
\hline & O2 C11 N1 121.41(11) & & $\mathrm{O} 2 \mathrm{C} 11 \mathrm{~N} 1122.07(12)$ \\
\hline & O3 C11 N1 109.83(10) & & O3 C11 N1 108.42(10) \\
\hline & O3 C12 C14 110.09(12) & & O3 C12 C15 101.53(12) \\
\hline & $\mathrm{O} 3 \mathrm{C} 12 \mathrm{C} 15$ 102.10(11) & & $\mathrm{O} 3 \mathrm{C} 12 \mathrm{C} 14$ 108.59(12) \\
\hline & $\mathrm{C} 14 \mathrm{C} 12 \mathrm{C} 15$ 111.13(16) & & $\mathrm{C} 15 \mathrm{C} 12 \mathrm{C} 14112.26(15)$ \\
\hline & O3 C12 C13 107.94(12) & & O3 C12 C13 109.59(10) \\
\hline & $\mathrm{C} 14 \mathrm{C} 12 \mathrm{Cl} 3113.50(16)$ & & $\mathrm{C} 15 \mathrm{C} 12 \mathrm{C} 13 \mathrm{111.02(15)}$ \\
\hline & $\mathrm{C} 15 \mathrm{C} 12 \mathrm{C} 13 \mathrm{111} .41(14)$ & & $\mathrm{C} 14 \mathrm{C} 12 \mathrm{C} 13$ 113.18(14) \\
\hline & C12 C13 H8 107.0(12) & & C12 C13 H8 110.7(11) \\
\hline & $\mathrm{C} 12 \mathrm{C} 13 \mathrm{H} 9$ 111.6(11) & & $\mathrm{C} 12 \mathrm{C} 13 \mathrm{H} 9107.7(11)$ \\
\hline & H8 C13 H9 108.4(16) & & H8 C13 H9 112.7(16) \\
\hline & $\mathrm{C} 12 \mathrm{C} 13 \mathrm{H} 10$ 107.8(13) & & $\mathrm{C} 12 \mathrm{C} 13 \mathrm{H} 10$ 111.2(13) \\
\hline & H8 C13 H10 110.0(18) & & H8 C13 H10 107.3(16) \\
\hline & H9 C13 H10 112.0(18) & & H9 C13 H10 107.2(17) \\
\hline & C12 C14 H11 107.5(17) & & C12 C14 H11 113.6(11) \\
\hline & C12 C14 H12 107.4(13) & & C12 C14 H12 112.0(12) \\
\hline & H11 C14 H12 111(2) & & H11 C14 H12 107.9(17) \\
\hline & C12 C14 H13 113.2(13) & & C12 C14 H13 109.9(13) \\
\hline & H11 C14 H13 110(2) & & H11 C14 H13 107.1(17) \\
\hline & H12 C14 H13 107.4(18) & & H12 C14 H13 105.8(16) \\
\hline & C12 C15 H14 109.9(14) & & C12 C15 H14 110(2) \\
\hline & C12 C15 H15 108.9(13) & & C12 C15 H15 111.0(15) \\
\hline & H14 C15 H15 112.7(19) & & H14 C15 H15 106(2) \\
\hline & C12 C15 H16 107.9(11) & & C12 C15 H16 110.0(14) \\
\hline & H14 C15 H16 108.2(18) & & H14 C15 H16 111(2) \\
\hline & H15 C15 H16 109.2(17) & & H15 C15 H16 108.3(19) \\
\hline
\end{tabular}


molecules. In contrast, in the case of the yellow crystal, the DPP skeleton and benzene rings are considerably twisted, and the distance between like molecules is large. In the case of the red crystal, it is clear that intermolecular interaction between the molecules is likely to occur in the $\pi$-conjugated portions. It is considered that the difference in the crystal structures is reflected in the difference in the colors of the two types of 272DPP-BOC.

\subsection{Fluorescence-Emission Characteristics}

It was revealed that the yellow and the red crystals also differ in terms of their fluorescence-emission characteristics. Fluorescence-emission spectra of the crystals in powder state are shown in Figure 5. The yellow crystal shows strong yellowish-green emission at $530 \mathrm{~nm}$ under excitation light at $470 \mathrm{~nm}$. On the other hand, the red crystal shows weak red-light emission at $625 \mathrm{~nm}$ under excitation light at $465 \mathrm{~nm}$. Moreover, the absolute quantum yield was $6.9 \%$ for the yellow crystals and $0.3 \%$ for the red ones. Generally, in a state in which molecules are gathered as a powder, concentration quenching occurs due to intermolecular interaction, so it is difficult to show intense fluorescence emission in such a state [9]. As shown by the results of the single-crystal X-ray structural analysis, compared to the yellow crystal, the red crystal has a shorter distance between the molecules. Consequently, concentration quenching due to intermolecular interaction is likely to occur in the case of the red crystal.

On the other hand, the yellow crystal has a large intermolecular distance, and the benzene-ring planes are arranged in various directions due to the twisting of the benzene-ring planes bonded to the DPP-skeleton plane. For that reason, it is considered that in the case of the yellow crystal the structure is such that intermolecular interaction in the $\pi$-conjugated-system portions is unlikely to occur. It is thus considered that intermolecular interactions are less likely to occur in the yellow crystal than in the red crystal; therefore, in the former case, concentration
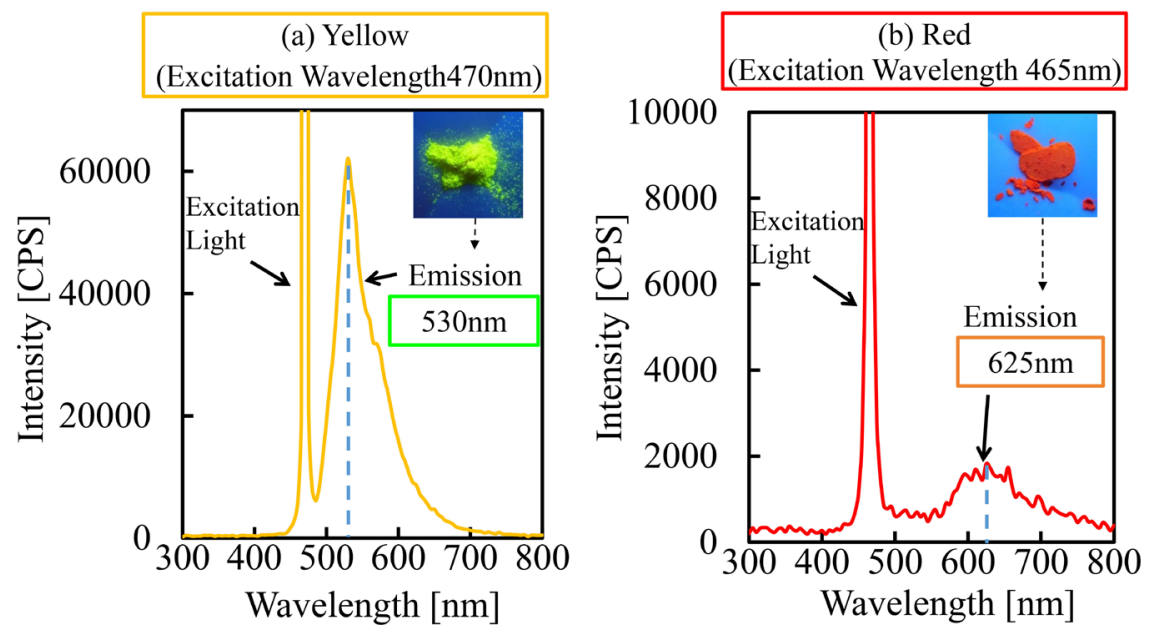

Figure 5. Solid-state fluorescence spectra of 272DPP-BOC Crystals. (a) 272DPP-BOC Yellow, (b) 272DPP-BOC Red. 
quenching does not occur so easily, and more intense fluorescence emission is exhibited.

\section{Conclusion}

A high-efficiency method for synthesizing 272DPP-BOC latent pigment by using MW irradiation was devised. By simply adding 272DPP and t-BOC reagent to NMP solvent and irradiating the mixture with microwaves for several seconds, 272DPP-BOC was obtained at high yield of $86.2 \%$. Yellow crystals and red crystals were obtained by recrystallization; however, single-crystal X-ray analysis revealed that the difference in colors is due to the difference in the crystal structures. In addition, the yellow crystal shows strong fluorescence emission at 530 $\mathrm{nm}$, but the red crystal shows weak light emission at $625 \mathrm{~nm}$. It is presumed that the cause of the different emissions comes from the type of overlapping of the molecules in the crystal structures.

\section{Conflicts of Interest}

The authors declare no conflicts of interest regarding the publication of this paper.

\section{References}

[1] Hervest, M. and Hunger, K. (2004) Industrial Organic Pigments. Wiley-VCH, Weinheim, 487-494.

[2] Nakazumi, H., (2004) Displays and Functional Dyes. CMC, Tokyo.

[3] Yanagisawa, H., Mizuguchi, J., Aramaki, S. and Sakai, Y. (2008) Organic field-effect transistor Devices Based on Latent Pigments of Unsubstituted Diketopyrrolopyrrole or Quinacridone. Japanese Journal of Applied Physics, 47, 4728-4731. https://doi.org/10.1143/JJAP.47.4728

[4] Suna, Y., Nishida, J., Fujisaki, Y. and Yamashita, Y. (2012) Ambipolar Behavior of Hydrogen-Bonded Diketopyrrolopyrrole-Thiophene Co-Oligomers Formed from Their Soluble Precursor. Organic Letters, 14, 3356-3359. https://doi.org/10.1021/ol3013364

[5] Kojima, H. and Mori, T. (2013) Estimated Mobility of Ambipolar Organic Semiconductors, Indigo and Diketopyrrolopyrrole. Chemistry Letters, 42, 68-70. https://doi.org/10.1246/cl.2013.68

[6] Zambounis, Z.., Hao, H. and Iqbal, A. (1997) Latent Pigments Activated by Heat. Nature, 388, 131. https://doi.org/10.1038/40532

[7] MacLean, E., Tremayne, M., Kariuki, B., Harris, K., Iqbal, A. and Hao, Z. (2000) Structural Understanding of a Polymorphic System by Structure Solution and Refinement from Powder X-Ray Diffraction Data: the $\alpha$ and $\beta$ Phases of the Latent Pigment DPP-BOC. Journal of the Chemical Society, Perkin Transactions, 2, 1513-1519. https://doi.org/10.1039/b001108h

[8] Mizuguchi, J. (2000) Correlation between Crystal and electronic Structures in Diketopyrrolopyrrole Pigments as Viewed from Exciton Coupling Effects. Journal of physical Chemistry $A, 104,1817-1821$. https://doi.org/10.1021/jp992302x

[9] Lunak Jr., S., Vala, M., Vynuchal, J., Ouzzane, I.., Horakova, P., Moziskova, P., Elias Z. and Weiter, M. (2011) Absorption and Fluorescence of Soluble Polar Diketo-Pyrrolo-Pyrroles. Dyes and Pigments, 91, 269-278. https://doi.org/10.1016/j.dyepig.2011.05.004 